\title{
FORMULATION, CHARACTERIZATION, AND IN VITRO EVALUATION OF NOVEL MICROEMULSION-BASED SPRAY FOR TOPICAL DELIVERY OF ISOTRETINOIN
}

\author{
ASHWINI WANI ${ }^{1 *}$, CHINTAN SANGHANI ${ }^{1}$, SWAPNIL WANI ${ }^{2}$ \\ ${ }^{1}$ Department of Quality Assurance, Vivekanand Education Society's College of Pharmacy, Chembur (E), Mumbai - 400 074, Maharashtra, \\ India. ²Department of Human Nutrition, BASF India Ltd., Turbhe, TTC Area, Navi Mumbai, Maharashtra, India. Email: ashwini.wani@ves.ac.in
}

Received: 02 April 2018, Revised and Accepted: 22 May 2018

\section{ABSTRACT}

Objective: The present study aimed at the development of a suitable formulation for the treatment of acne using microemulsion-based topical spray system. In this study, we have designed and evaluated novel isotretinoin microemulsion-based spray and gel using basil oil. The rationale of work is to develop single topical formulation with multiple effects by combining effect of retinoid (prevention of comedones formation) and natural oil like basil oil (antibacterial, anti-inflammatory, and penetration enhancer).

Methods: The optimized microemulsion-based spray comprised basil oil, Kolliphor RH40, Ethanol, and water was prepared by an aqueous titration method with the help of a ternary phase diagram. The microemulsion-based gel was prepared using Carbopol ETD 2020. The formulations were evaluated for their physicochemical properties. The formulations were subjected to antibacterial activity against Propionibacterium acnes, Staphylococcus epidermidis, and Staphylococcus aureus using cup plate method against hydrogen peroxide as standard. Finally, the drug released from spray and gel formulations was studied and compared to a commercially available product.

Results: Isotretinoin microemulsion-spray showed highest flux $\left(27.67 \pm 0.12 \mu \mathrm{g} / \mathrm{cm}^{2} / \mathrm{h}\right)$ in comparison to formulated microemulsion gel $\left(21.81 \pm 0.103 \mu \mathrm{g} / \mathrm{cm}^{2} / \mathrm{h}\right)$ and conventional marketed gel $\left(19.29 \pm 0.34 \mu \mathrm{g} / \mathrm{cm}^{2} / \mathrm{h}\right)$. The particle size of optimized microemulsion was found to be $68.43 \mathrm{~nm}$. The results indicate that the formulated microemulsion-based spray presents a promising alternative for acne therapy.

Conclusion: This mentioned delivery system presented several benefits such as photostability, better patient compliance, antibacterial activity, and good penetration into the desired skin layer when compared to in vitro diffusion of conventional gel and microemulsion-based gel.

Key words: Acne, Isotretinoin, Microemulsion spray, Natural oil, Antibacterial activity.

(C) 2018 The Authors. Published by Innovare Academic Sciences Pvt Ltd. This is an open access article under the CC BY license (http://creativecommons. org/licenses/by/4. 0/) DOI: http://dx.doi.org/10.22159/ajpcr.2018.v11i10.27019

\section{INTRODUCTION}

Acne is a common skin disease that nearly affects $80 \%$ of the population at some point in their lifetime [1]. It is associated with sebaceous follicle and starts appearing after the onset of puberty and can extend up to $40-50$ years of age [2]. Although acne can sometimes be a cosmetic concern, it can also be disfiguring and scarring. Propionibacterium acnes is thought to be the culprit bacteria contributing to acne. Common firstline treatment for acne is a topical combination of benzoyl peroxide, retinoid, and antibiotic [3].

Topical therapy is the first line of therapeutic treatment for acne. Topical retinoids such as isotretinoin, adapalene, and tazarotene have proven to be effective. These retinoids inhibit the microcomedone formation and reduce the non-inflammatory and inflammatory lesions [4-6]. From these three drugs, isotretinoin is found to be extremely effective which suppresses acne over the long term. It is used for the treatment of severe acne and other dermatological diseases. The irritation of topical isotretinoin therapy is the limiting factor in maximizing treatment because of high lipophilic (log $\mathrm{P}=4.20$ ) nature of the drug. It tends to accumulate in the upper stratum corneum, thereby delaying penetration into the lower skin layers.

In recent years, scientists reviewed microemulsions as a potential carrier of topical anti-acne drug delivery [7]. It is noteworthy that in topical and transdermal formulations they improve biopharmaceutical properties by enhancing drug permeation through stratum corneum and then to the deeper tissues. This property may be related to several different mechanisms such as the presence of surfactants and cosurfactants acting as absorption enhancers and good wetting ability [8].

The microemulsion formulations were aimed to deliver in the form of a non-aerosol spray dosage form. The motive behind using a spray delivery was to ensure site-specific delivery of drug to enhance the diffusion through skin. Furthermore, it provides an advantage of ease of application on the different affected body area, hence increasing patient compliance.

Moreover, a combination of herbal oil, having antimicrobial activity, with isotretinoin will inhibit the growth of colonized $P$. acnes bacteria around the acne infected skin cells. The purpose of using antibacterial agents is not only to kill the acne causing bacteria but also to prevent the formation of new lesions and providing relief from inflammation. The unaffected skin cells surrounding the acne do not get infected by the bacterium by the presence of antimicrobial herbal oil. This asset of using herbal oil can be utilized to treat not only acne on the face but also on the acne on the back, chest, or shoulders as well. This gives the additional advantage in acne treatment along with isotretinoin.

Such herbal oil can be used as an oily phase in the microemulsion, hence playing a role of vehicle for the drug by solubilizing it, possessing antimicrobial activity and reducing the inflammation caused by acne and by providing a cooling sensation to the skin.

In this research article, we present two novel microemulsion-based systems for topical delivery of isotretinoin. Both microemulsion-based spray (MBS) and microemulsion-based gel (MBG) were studied in terms 
of physicochemical properties with a special focus on antibacterial properties. Moreover, the obtained formulations were tested in vitro, and the results were compared with that of the commercial topical gel. The aim of this study was to analyze in detail the factors that affect the properties of the final product and key considerations to be taken in the new topical formulation design process.

\section{MATERIALS AND METHODS}

\section{Materials}

Isotretinoin was obtained as a gift sample from Cipla Ltd., India, while Kolliphor RH40 sample as procured from BASF, India. Basil oil was purchased from Shree Siddhi Aroma, India. Carbopol ETD 2020 was obtained as a gift sample from Lubrizol, India.

\section{Formulation of microemulsion}

Screening of essential oils, surfactants, and cosurfactants for microemulsion

The solubility of isotretinoin in various oils, surfactants, and cosurfactants was determined to find out the appropriate oils, surfactants, and cosurfactants with good solubilizing capacity. Oils employed were known to have an antibacterial activity such as eucalyptus oil, neem oil, peppermint oil, basil oil, and lemon oil. Surfactants employed in this study were Tween 80, Span 80, Span 20, Kolliphor RH240, and Phospholipid 90G while cosurfactants used were Transcutol P, isopropyl alcohol, ethanol, PEG 400, propanol, and butanol. An excess amount of isotretinoin was added to $5 \mathrm{~mL}$ of each oil, surfactant, and cosurfactant. The resultant mixture was shaken on rotary flask shaken for $48 \mathrm{~h}$ at ambient temperature followed by centrifugation for $10 \mathrm{~min}$ at $6000 \mathrm{rpm}$. The supernatant was filtered through a membrane filter, and isotretinoin was determined spectrophotometrically at $344 \mathrm{~nm}$ [9].

\section{Emulsification studies}

To know the ideal combination of surfactant/cosurfactant and their ratio, with selected basil oil, emulsification studies of surfactants (Tween 80, Kolliphor RH40, and Phospholipid 90G), and cosurfactants (Transcutol P, ethanol, PEG 400, and propanol) were conducted.

A premix of surfactant mixture $\left(\mathrm{S}_{\text {mix }}\right)$ was prepared by mixing surfactant and cosurfactant taken in the ratios of $1: 1,2: 1$, and $3: 1$ by vortex mixing. Basil oil was added to each $S_{\text {mix }}$ ratio in the ratio $1: 1$ and was vigorously vortexed. These oily mixtures were titrated against distilled water at ambient temperature. Amount of water required to convert the oily mixture from clear to turbid was considered as an endpoint. The $S_{\text {mix }}$ combination and ratio which showed maximum incorporation of water was considered the best emulsifying mixture for basil oil.

\section{Construction of pseudoternary phase diagrams}

Based on emulsification data, following $\mathrm{S}_{\text {mix }}$ were screened for ternary phase diagrams as shown in Table 1. For each phase diagram, oil and specific $S_{\text {mix }}$ ratio were mixed vigorously in different volume ratios from $0: 10$ to 10:0 under gentle agitation. Ternary phase diagrams were developed using the aqueous titration method. Slow titration with the aqueous phase was done for each combination of oil and $\mathrm{S}_{\text {mix }}$ separately. The quantity of aqueous phase-added was speckled to produce a water concentration in the range of $5-95 \%$ of total volume at around 5\% intervals. After each 5\% addition of the aqueous phase to the oil, visual observation was made and recorded. Water was being added to the lipid mixture until the endpoint was achieved, endpoint being a conversion of a clear solution to turbid. Usually, after the clear mixture became turbid at a certain point (beginning of phase inversion area), the turbid mixture turned to be clear (beginning of $\mathrm{O} / \mathrm{W} \mathrm{ME}$ area) and, then, finally turbid once again (end of $0 / \mathrm{W}$ microemulsion area) with the continuous addition of water [10].
Table 1: $S_{\text {mix }}$ ratio for ternary phase diagrams

\begin{tabular}{llll}
\hline Oil & Surfactant & Cosurfactant & Ratio \\
\hline Basil oil & Tween 80 & Ethanol & $3: 1$ \\
Basil oil & Tween 80 & Propanol & $3: 1$ \\
Basil oil & Kolliphor RH40 & Ethanol & $3: 1$ \\
\hline
\end{tabular}

\section{Preparation of MBS}

After selecting the combination of excipients providing greater emulsification and larger area in the phase diagram, calculated amount of drug was incorporated in the lipid carrier, followed by addition of S/Co-S mixture in a fixed proportion to produce a clear mixture. Then, defined amount of water was added and mixed by vortex mixer until clear, isotropic, transparent solution is formed. The fluidic microemulsion was intended to be used as a topical spray.

\section{Optimization of MBS formulation}

Optimization was carried out to identify the parameters which will result in MBS with minimum particle size and the maximum amount of drug diffused. A 2-factor 3-level full factorial design $\left(3^{2}\right)$ was used for the optimization of isotretinoin loaded microemulsion with two independent variables at three different levels. The different levels of each variable are indicated in Table 2.

\section{Evaluation of MBS formulation [11-15]}

The MBS was evaluated on various parameters like.

\section{Appearance}

The microemulsions were checked for optical transparency and homogeneity by visually observing against the light. The monophasic systems were also checked for the presence of any undissolved drug or solid particulates.

Particle size

The average droplet size and polydispersity index of microemulsion were measured by photon correlation spectroscopy with in-built zetasizer.

Zeta potential

The assessment of the physical stability of colloidal dispersions is characterized by measuring zeta potential of microemulsions. This was measured using Malvern Zetasizer.

\section{Viscosity}

The viscosity of microemulsion was measured using a Brookfield viscometer with spindle LV - S63.

$\mathrm{pH}$

pH was determined using $\mathrm{pH}$ meter.

Drug content

Formulation assay was conducted by spraying an amount of formulation equivalent to the dose in a volumetric flask. The drug was extracted using methanol, filtered and appropriate dilutions were made and further analyzed by ultra violet (UV) spectrophotometer.

\section{Spray uniformity}

Metered dose topical spray solution was tested by holding the dose leaving from the actuator of the atomizing device. The container was shaken for 5 $\mathrm{s}$, and the initial dose was taken as a trial. The next immediate dose was fired into the volumetric flask. The drug was extracted from methanol. The content of drug in the collective rinses was determined by evaluating the content uniformity at $4^{\text {th }}, 12^{\text {th }}, 26^{\text {th }}, 48^{\text {th }}, 72^{\text {nd }}$, and $88^{\text {th }}$ shots [12].

\section{Formulation of MBG}

Preparation of isotretinoin $M B G$

Isotretinoin microemulsion gel was prepared using Carbopol ETD 2020. Carbopol was directly added to the prepared microemulsion 
Table 2: $3^{2}$ full factorial design used for Optimization

\begin{tabular}{lllllll}
\hline Levels (coded values) & Actual values & & Response \\
\cline { 2 - 3 } & $\mathbf{S}_{\text {mix }}$ concentration (\%) $\left(\mathbf{X}_{\mathbf{1}}=\mathbf{A}\right)$ & Lipid concentration (\%) $\left(\mathbf{X}_{2}=\mathbf{B}\right)$ & & $\mathbf{Y}_{\mathbf{1}}$ & $\mathbf{Y}_{2}$ \\
\hline Low (-1) & 30 & 8 & & Particle size (nm) & Drug diffused (\%) \\
Intermediate (0) & 40 & 12 & & & \\
High (+1) & 50 & 16 & & & \\
\hline
\end{tabular}

slowly with continuous stirring with the help of the overhead stirrer. The dispersion was neutralized using triethanolamine to obtain a gel.

\section{Evaluation of $M B G$ formulation}

The MBG was evaluated on various parameters like.

Appearance

The gels were checked for its organoleptic properties such as color, odor, clarity, and rheological behavior such as its translucency and homogeneity.

Viscosity measurement

Viscosity was measured using a Brookfield viscometer LVDV-IIIU employing spindle S92 (T-shaped spindle) rotated at $5 \mathrm{rpm}$. All aspects of testing were controlled using Rheocalc Software.

Measurement of $\mathrm{pH}$

The $\mathrm{pH}$ value was determined using a digital $\mathrm{pH}$ meter which was standardized using $\mathrm{pH} 4$ and 7 buffer before use. A weighed the quantity of $0.1 \mathrm{~g}$ of gel was diluted in $10 \mathrm{ml}$ of distilled water. Then, the $\mathrm{pH}$ of the aqueous solution of the gel was measured.

\section{Spreadability}

The spreadability of the gel was determined by placing $1 \mathrm{~g}$ gel within a pre-marked circle of $1 \mathrm{~cm}$ diameter on a glass plate over which a second glass plate was placed. A weight of $20 \mathrm{~g}$ was allowed to rest on the upper glass plate for $5 \mathrm{~min}$. The increase in the diameter due to spreading of the gels was noted, and the mean diameter was taken.

Drug content

Drug content of the gel was determined by UV spectroscopy. Accurately weighed $1 \mathrm{~g}$ of the formulation was transferred to $10 \mathrm{ml}$ volumetric flask. Methanol was added and sonicated for $10 \mathrm{~min}$ for complete extraction. Further, dilutions were made with methanol and absorbance was measured at $344 \mathrm{~nm}$ by UV spectroscopy.

\section{In vitro diffusion profile of MBS and MBG}

Diffusion protocol was finalized by doing preliminary studies of $\mathrm{pH}-$ dependent solubility and preparation of calibration curve in selected diffusion media. The diffusion experiment was performed using Franz diffusion cell fitted with egg membrane [16]. The effective permeation area was $3.14 \mathrm{~cm}^{2}$ (20 mm diameter), and the receptor compartment was filled with $10 \mathrm{ml}$ of diffusion media of phosphate buffer $\mathrm{pH} 7.2$ and ethanol in the ratio of $60: 40 \mathrm{v} / \mathrm{v}$. The temperature of the diffusion media in the receptor compartment was controlled at $37 \pm 1^{\circ} \mathrm{C}$. Formulation ( $1 \mathrm{~g}$ ) was gently placed in the donor chamber, and then the diffusion cell was covered with aluminum foil to prevent light exposure. At 15, 30, 45, 60, 120, 240, and 360 min, an aliquot of $2 \mathrm{ml}$ was withdrawn from receptor compartment and replaced immediately with an equivalent amount of diffusion media to maintain sink conditions. The concentration of isotretinoin in receptor fluid was analyzed by UV spectroscopy at $340 \mathrm{~nm}$, and a cumulative amount of drug diffused was calculated after each time interval.

\section{Determination of Diffusion Kinetics}

The in vitro drug diffusion data obtained which were fitted into kinetic models, namely Zero-order (a), First-order (b), Higuchi (c) and Korsmeyer-Peppas, and (d) to know the pattern of drug permeation.

\section{In vitro antibacterial activity of MBS and MBG}

In vitro antibacterial activity was done to study the effectiveness of basil oil as an antibacterial agent in isotretinoin microemulsion. The antibacterial efficacy of formulated microemulsion was evaluated against Staphylococcus aureus (MTCC No. 6538), Staphylococcus epidermidis (MTCC No. 3615), and P. acnes (MTCC No. 1951) using cup plate method and bacterial concentration used was $3 \times 108 \mathrm{CFU} / \mathrm{ml}$. The samples which were introduced into the cup were ME formulation and control placebos of basil oil (8\%), ethanol (7.5\%), and ISTN $(0.05 \%)$ on four different plates. The plates were incubated at $37^{\circ} \mathrm{C}$ for $48 \mathrm{~h}$ under anaerobic conditions for $P$. acnes and aerobic condition for other bacteria.

For control plates, each of positive and negative control was prepared. The entire cup plate tests were performed in triplicate on each organism and antibacterial activity was expressed as zone of inhibition in $\mathrm{mm}$. The zone of inhibition for optimized ME formulation against standard benzoyl peroxide was measured.

\section{Photostability study of isotretinoin in MBS and MBG}

The photostability of isotretinoin was assessed by recording its absorption spectra over the wavelength range of $200-400 \mathrm{~nm}$ in two matched quartz cells with a $1 \mathrm{~cm}$ light path using a double beam UV-visible spectrophotometer at the following conditions: Slow scan speed, time response of $1 \mathrm{~s}$, and a spectral band of $1 \mathrm{~nm}$. The radiant power was adjusted to the lower value in the instrumental scale and the cabinet temperature at $25^{\circ} \mathrm{C}$. These gentle experimental conditions were set because of the high sensitivity of the drug to light, allowing so to obtain more accurate control of the photodegradation process [17]. Methanolic solution of isotretinoin $(25 \mu \mathrm{g} / \mathrm{mL})$ was exposed to natural sunlight, and UV spectra of all the samples were recorded just after preparation $(\mathrm{t}=0)$ and at time intervals of 15,30 , $60,90,120,150,180,210$, and 240 min after suitable dilution with methanol.

The formulations were also exposed to light under the same experimental conditions described above for solution, and recording of spectra was done at the same irradiation times. For these formulations, spectrophotometric measurements were performed by diluting it suitably with methanol. Baseline correction was done using a plain microemulsion dispersion diluted suitably with methanol to nullify any possible absorption arising from the excipients. Sufficient care was taken to maintain similar experimental conditions for both the samples, i.e., isotretinoin in methanol and isotretinoin in the microemulsion. The degradation was evaluated on the basis of kinetic photodegradation constant $\mathrm{k}$ and half-life time $(\mathrm{t} 1 / 2)$ with respect to the initial percent absorbance.

\section{RESULTS}

\section{Formulation of microemulsion}

Screening of essential oils, surfactants, and cosurfactants for microemulsion

Solubility studies were mainly carried out for identifying suitable excipients for development of microemulsion of isotretinoin. Screening of various excipients such as essential oils, surfactants, and 
cosurfactants was carried out based on solubility, and the results are shown in Table 3.

Basil oil showed maximum solubilization of isotretinoin as compared to the remaining oils in which the drug was less soluble. Similarly, Tween 80 showed greater solubility as compared to meager solubility shown by Kolliphor RH40 and Phospholipon 90 G. It was observed that in cosurfactants short chain, unbranched alcohols such as ethanol, propanol, and butanol showed better solubility than other class of solvents such as Transcutol P and PEG 400.

\section{Emulsification studies}

Based on the maximum incorporation of water and formation of clear, homogenous and translucent systems of basil oil, three emulsifying mixtures were screened. These were Tween 80/ethanol, Tween 80/ propanol, and Kolliphor RH40/ethanol each in the ratio 3:1. The results obtained from emulsification studies indicated that Kolliphor RH40 along with ethanol showed a good efficiency and water solubilizing capacity in the presence of basil oil.

\section{Construction of pseudoternary phase diagrams}

The pseudoternary phase diagrams were constructed to determine the concentration range of components in the existence range of microemulsion. It was seen that Basil oil was solubilized completely with Kolliphor RH40 and Tween 80 but the Wmax of Basil oil/Kolliphor RH40/ Ethanol mixtures were higher as compared to Basil oil/Tween 80/Ethanol and Basil oil/Tween 80/Propanol (although not statistically significant). Fig. 1 shows the pseudoternary phase diagrams for all three mixtures.

The prepared microemulsions were formed spontaneously when a pre-defined amount of water was added at once to the oil and $S_{\text {mix }}$ mixture with continuous stirring. The same was also seen in the presence of isotretinoin $(0.05 \% \mathrm{w} / \mathrm{w})$ which was added to the oil phase of the system. No deviations were found in the pre-defined amount of water while preparing the microemulsion with the drug from that of microemulsion without the drug, i.e., inclusion of drug had no impact on the phase boundary of the region of a pseudoternary phase diagram.

\section{Formulation of MBS}

From the phase diagram studies, microemulsions were formulated using $\mathrm{S}_{\text {mix }}$ as Kolliphor RH40 and ethanol in the ratio 3:1 having basil oil as a lipid phase and distilled water as the aqueous phase. The microemulsions loaded with isotretinoin were of low viscosity, fluidic, yellow colored translucent and clear solution.

\section{Optimization of MBS}

For the present study, the criteria for optimum batch were decided as the one which shows minimum particle size and maximum percentage of drug diffusion (Table 4). Based on the response surface model design batches and their response $s$, three solutions were suggested by Design-Expert ${ }^{\circledast}$ (Table 5). The optimized formula that was used for the formulation is represented in Table 6. It is found that the desirability value of Batch M9 is closer to unity. Hence, the formulation M9 is considered the optimized formulation.

\section{Evaluation of MBS}

Various parameters such as appearance, particle size, zeta potential, viscosity, $\mathrm{pH}$, drug content, and spray uniformity were evaluated, and the results are depicted in Table 7.

\section{Formulation of $M B G$}

The MBG was prepared using the optimized microemulsion system (M9). The gel was prepared using Carbopol ETD 2020 as a gelling agent. Table 8 shows the final formula for MBG. The MBG was characterized and evaluated for its various parameters and compared with the marketed gel of isotretinoin.

The formulation was evaluated based on various parameters such as appearance, viscosity, $\mathrm{pH}$, spreadability, drug content, and percent drug diffused and the results obtained were compared with marketed gel as shown in Table 9.

\section{In vitro diffusion profile of MBS and MBG}

Solubility values of various buffers in varied ratios with ethanol were estimated, and the results are depicted in Fig. 2. Based on solubility profile phosphate buffer 7.2 was selected with 6:4 ratio with ethanol as per solubility of API. The calibration curve of the drug in this buffer media was linear with $\mathrm{R}^{2}=0.9988$. The cumulative release of marketed formulation, optimized microemulsion formula (M9) of spray and microemulsionbased gel of M9 were compared against time, as shown in Fig. 3.

\section{Determination of diffusion kinetics}

Table 10 represents linearity $\left(R^{2}\right)$, and diffusion component (n) values obtained from the kinetic plots of in vitro drug diffusion studies.

Table 3: The solubility profiles of isotretinoin in various excipients

\begin{tabular}{llllll}
\hline Surfactants & Concentration $(\mathbf{m g} / \mathbf{m l})$ & Co-surfactants & Concentration $\mathbf{( m g} / \mathbf{m l})$ & Herbal oils & Concentration $(\mathbf{m g} / \mathbf{m l})$ \\
\hline Tween 80 & 88.28 & Transcutol P & 50.66 & Eucalyptus oil & 69.25 \\
Span 80 & 11.73 & IPA & 42.07 & Neem oil & 16.28 \\
Span 20 & 13.24 & Ethanol & 114.79 & basil oil & 77.84 \\
Kolliphor RH40 & 62.07 & PEG 400 & 28.32 & Peppermint oil & 37.65 \\
Phospholipid 90G & 25.31 & Propanol & 84.51 & Lemon oil & 50.84 \\
& & Butanol & 69.03 & & \\
\hline
\end{tabular}

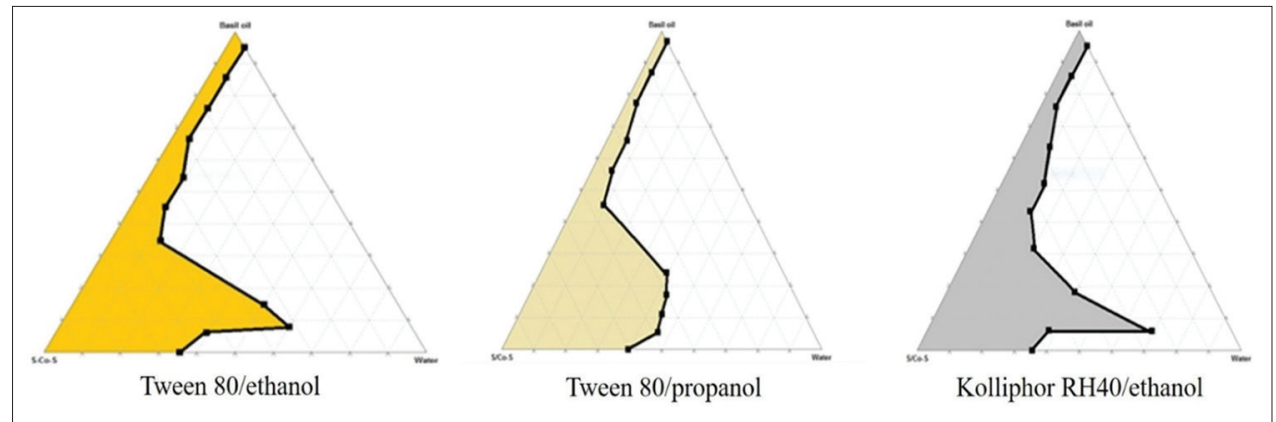

Fig. 1: Pseudoternary phase diagrams 


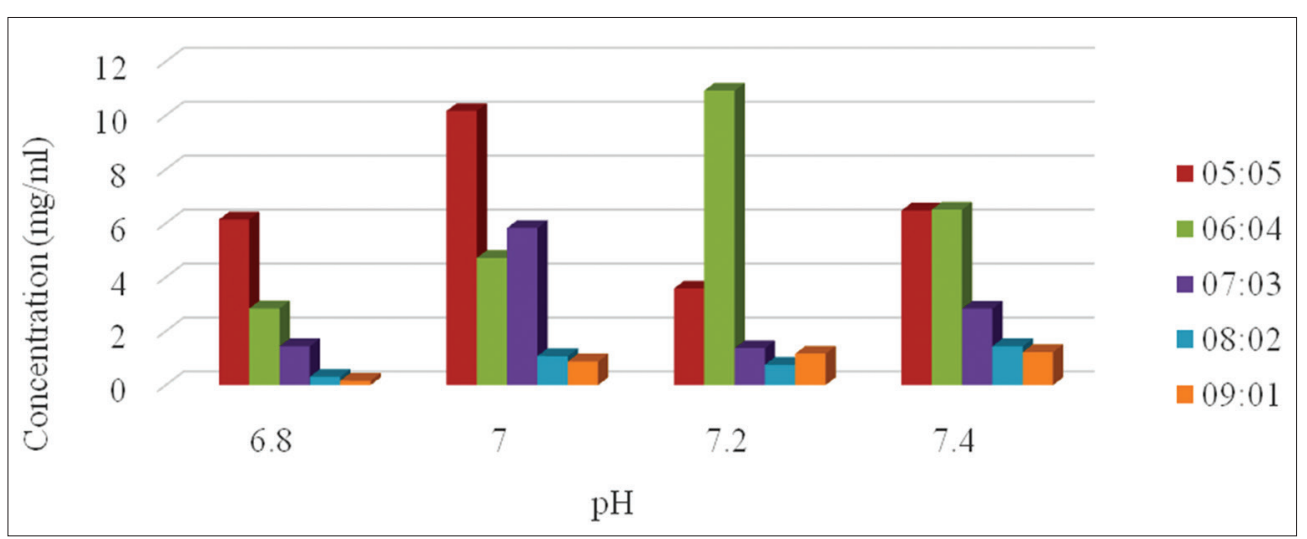

Fig. 2: pH-dependent solubility

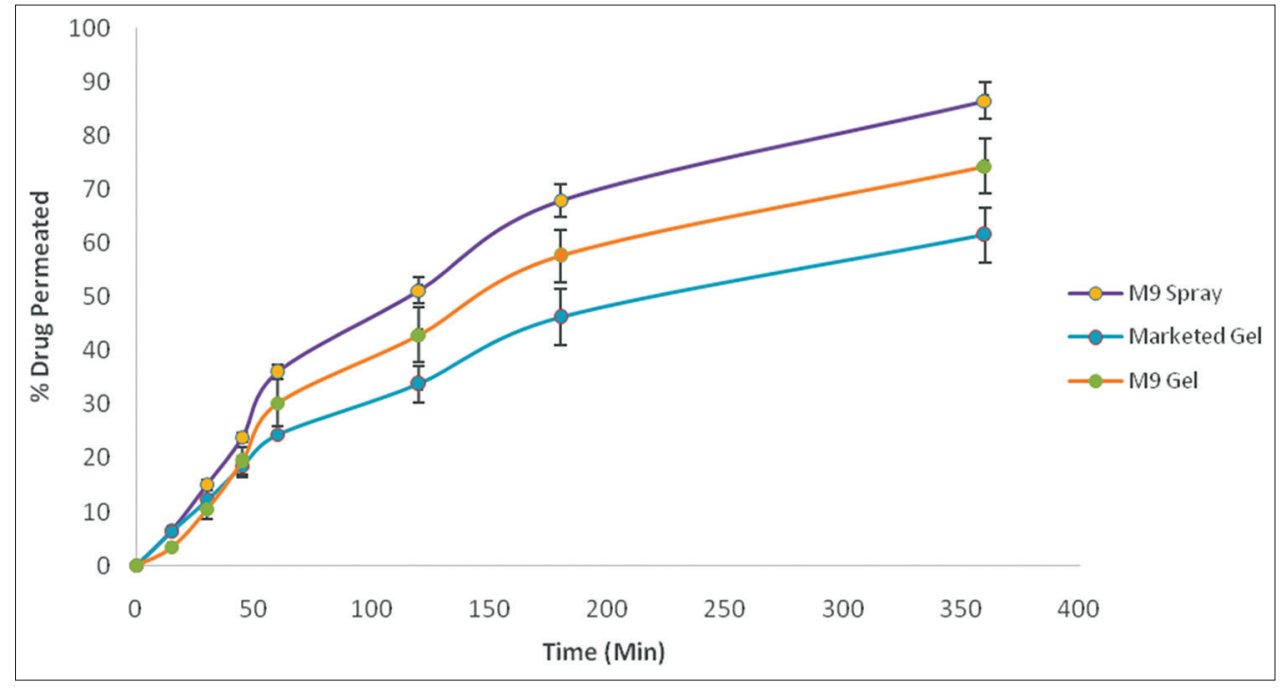

Fig. 3: Comparison of in vitro diffusion profile

Table 4: Optimization of microemulsion batch

\begin{tabular}{|c|c|c|c|c|c|c|c|}
\hline \multirow[t]{2}{*}{ Runs } & \multirow[t]{2}{*}{ Batch code } & \multicolumn{2}{|c|}{ Surfactant mix concentration $(\%)$} & \multicolumn{2}{|c|}{ Lipid concentration (\%) } & \multicolumn{2}{|l|}{ Dependent variable } \\
\hline & & Coded & Actual & Coded & Actual & Particle size (nm) & Drug diffused (\%) \\
\hline 1 & M1 & +1 & 50 & +1 & 16 & 331.1 & 41.02 \\
\hline 2 & M2 & 0 & 40 & +1 & 16 & 81.79 & 63.91 \\
\hline 3 & M3 & -1 & 30 & +1 & 16 & 287.1 & 19.25 \\
\hline 4 & M4 & +1 & 50 & 0 & 12 & 202.8 & 34.42 \\
\hline 5 & M5 & 0 & 40 & 0 & 12 & 161.2 & 37.31 \\
\hline 6 & M6 & -1 & 30 & 0 & 12 & 143.7 & 20.62 \\
\hline 7 & M7 & +1 & 50 & -1 & 8 & 163.2 & 21.22 \\
\hline 8 & M8 & 0 & 40 & -1 & 8 & 251.3 & 31.26 \\
\hline 9 & M9 & -1 & 30 & -1 & 8 & 68.79 & 86.43 \\
\hline
\end{tabular}

Table 5: Three solutions for optimized batch were suggested by Design-Expert ${ }^{\circledast}$

\begin{tabular}{lllllll}
\hline No. & Concentration of surfactant & Concentration of lipid & Particle size & \% Drug diffused & Desirability & Selected \\
\hline 1. & 30.0 & 8.0 & 116.24 & 68.41 & 0.952 & 0.948 \\
2. & 30.15 & 7.91 & 141.3 & 63.27 & 0.926 & \\
3. & 30.0 & 8.11 & 146.3 & 60.32 & & \\
\hline
\end{tabular}

The in vitro release profiles of the drug from the formulation could be best expressed by Higuchi model as the plots showed the highest linearity $\left(\mathrm{R}^{2}=0.9735\right)$ than the other plots.

\section{In vitro antibacterial activity}

The in vitro antibacterial activity of the microemulsion containing $8 \%$ basil oil was determined by growth inhibition zones against $P$. acnes,
S. epidermidis, and S. aureus. Zone of inhibition of benzoyl peroxide, which is commonly used as an antibiotic in the treatment of acne, was also found out as a standard to compare the effectiveness of developed formulation. The results are ically represented in Fig. 4.

From the zone of inhibition data, it was clearly observed that inhibition took place significantly due to basil oil. A marked difference was 
Table 6: Optimized formula

\begin{tabular}{ll}
\hline Ingredients & Quantity taken (\%) \\
\hline Isotretinoin & 0.05 \\
Basil oil & 8 \\
Kolliphor RH40 & 22.5 \\
Ethanol & 7.5 \\
Distilled water & 61.95 \\
\hline
\end{tabular}

Table 7: Evaluation of microemulsion spray

\begin{tabular}{lll}
\hline S. No. & Parameters & Results \\
\hline 1. & Appearance & $\begin{array}{l}\text { Clear, isotropic, and } \\
\text { transparent yellow solution }\end{array}$ \\
& & W/O type \\
2. & Dilutabilty test & $68.79 \mathrm{~nm}$ \\
3. & Particle size & $86.43 \%$ \\
4. & Percent drug release & $108 \pm 4.725 \mathrm{cP}$ \\
5. & Viscosity & 0.270 \\
6. & Poly dispersibility index & $-0.403 \pm 0.014 \mathrm{mV}$ \\
7. & Zeta potential & $6.25 \pm 0.088$ \\
8. & pH & \\
\hline
\end{tabular}

Table 8: Optimized formula for MBG

\begin{tabular}{ll}
\hline Ingredients & Quantity taken (\%) \\
\hline Carbopol@ 2020 ETD NF & 0.75 \\
Isotretinoin & 0.05 \\
Basil oil & 8 \\
Kolliphor RH40 & 22.5 \\
Ethanol & 7.5 \\
Triethanolamine $(50 \% \mathrm{w} / \mathrm{w})$ & q.s. \\
Distilled water & 61.2 \\
\hline
\end{tabular}

MBG: Microemulsion-based gel observed in the zones of microemulsion formulation and benzoyl peroxide standard against all three species, formulation bettering the latter. It was also seen that the zone of inhibition of microemulsion formulation was lesser than the placebo of basil oil. This may be attributed as basil oil placebo is itself an oil phase (in DMSO), which allows faster diffusion as compared to the ME formulation, in which basil oil is bonded with an aqueous phase which retards the diffusion comparatively. Thus, the zone of inhibition achieved was predominantly due to the presence of basil oil in the formulation.

\section{Photostability studies}

The photochemical reaction in methanol solution demonstrated a rapid isomerization of isotretinoin followed by a further degradation consisting in the minimization of the absorbance peaks. Therefore, the absorbance values of these maxima were used to evaluate the kinetics of the photodegradation processes [18]. Isotretinoin degradation curves plotted in Fig. 5 summarizes the degradation kinetic parameters.

Through photostability studies, the formulation demonstrated an increase in isotretinoin half-life to about 13 times in comparison with amethanolic solution, under direct sunlight.

\section{CONCLUSION}

In this study, novel microemulsion-based spray with isotretinoin as a potential topical drug delivery system was successfully designed and evaluated. The designed microemulsion-based spray system differed from the lab-scale microemulsiongel and commercial product containing isotretinoin gel in terms of drug diffusion. The particle size of optimized formulation was quite low, and consequently, permeation of drug was greatly enhanced when isotretinoin was loaded in microemulsion when compared to marketed gel. The microemulsion has added advantage of antibacterial action on acne-causing bacteria over the conventional gel due to the presence of basil oil, increased photostability of the drug due

Table 9: Results of ME-based gel compared with marketed gel

\begin{tabular}{llll}
\hline S. No. & Parameters & Evaluation & Marketed gel \\
\cline { 3 - 4 } & & MBG & Transparent, translucent, yellow gel \\
\hline 1. & Appearance & Transparent, translucent, yellow gel & $5750 \pm 11.257 \mathrm{cP}$ \\
2. & Viscosity & $6800 \pm 9.35 \mathrm{cP}$ & $6.4 \pm 0.045$ \\
3. & pH & $6.5 \pm 0.02$ & $5.9 \pm 0.02 \mathrm{~cm}$ \\
4. & Spreadability & $7.2 \pm 0.01 \mathrm{~cm}$ & $99.21 \pm 0.59$ \\
5. & Drug content (\%) & $98.19 \pm 0.78$ & $61.53 \pm 1.33$ \\
6. & Percent drug diffused & $75.34 \pm 1.02$ & \\
\hline
\end{tabular}

All the values in results are expressed as mean \pm SEM $(n=3)$. SEM: Standard error mean, MBG: Microemulsion-based gel

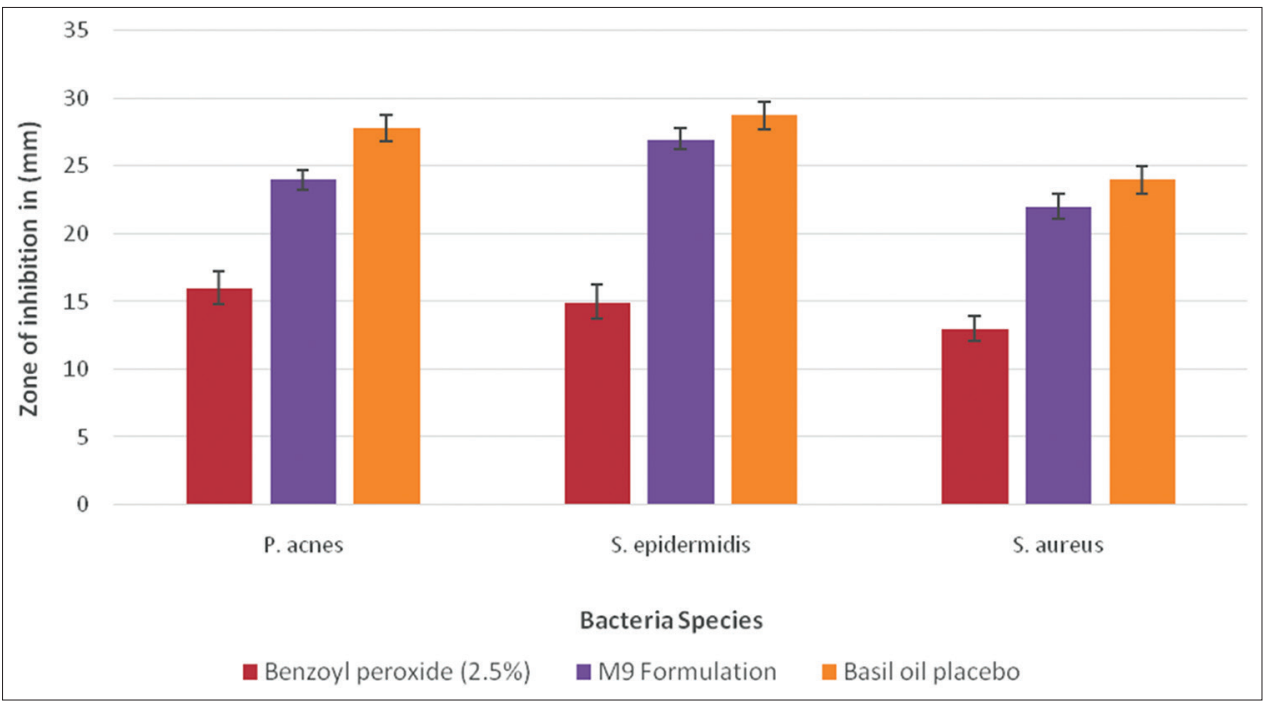

Fig. 4: In vitro antibacterial activity 
Table 10: Linearity $\left(\mathrm{R}^{2}\right)$ and diffusion component (n) values from the kinetic plots of the in vitro drug diffusion studies

\begin{tabular}{|c|c|c|c|c|c|}
\hline Model & Zero-order & First-order & Higuchi model & \multicolumn{2}{|c|}{ Korsmeyer-Peppas model } \\
\hline $\mathrm{R}^{2}$ & 0.8901 & 0.5943 & 0.9735 & 0.9557 & $\begin{array}{l}\text { Diffusion component }(\mathrm{n}) \\
0.9442\end{array}$ \\
\hline
\end{tabular}

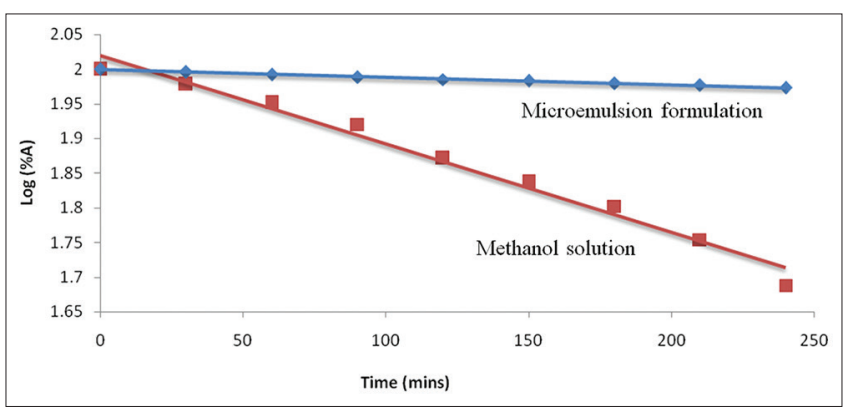

Fig. 5: Photodegradation plots of isotretinoin in methanol solution and microemulsion

to incorporation in the microemulsion, better patient compliance due to ease of application and better penetration into the desired skin layer.

\section{AUTHOR'S CONTRIBUTIONS}

The first author has provided study conception and design of work and served as a mentor. The second author has carried out the research. Both the first and second author has drafted the manuscript and carried critical revision. The third author has provided samples for research and assisted in the design of work.

\section{CONFLICTS OF INTEREST}

The authors declare that they have no conflicts of interest.

\section{REFERENCES}

1. Dunn JA, Coburn RA, Evans RT, Genco RJ, Walters, KA. Novel topically active antimicrobial/anti-inflammatory compounds for acne. In: Walters KA, Roberts MS, editors. Dermatologic, Cosmeceutic and Cosmetic Development. New York: Informa; 2008. p. 243-54.

2. Prasad SB. Acne vulgaris: A Review on pathophysiology and treatment. Asian J Pharm Clin Res 2016;9:54-9.

3. Zaenglein AL, Pathy AL, Schlosser BJ, Alikhan A, Baldwin HE,
Berson DS, et al. Guidelines of care for the management of acne vulgaris. J Am Acad Dermatol 2016;74:945-73.

4. Johnson BM, Nunley JR. Use of systemic agents in the treatment of acne vulgaris. Am Fam Phys 2000;61:1823-30.

5. Nikam S. Anti-acne gel of isotretinoin: Formulation and evaluation. Asian J Pharm Clin Res 2017;10:257-66.

6. Toyodo M, Morohashi M. An overview of topical antibiotics for the treatment of acne. Dermatol 1998;196:130-4.

7. Talegaonkar S, Azeem A, Ahmad FJ, Khar RK, Pathan SA, Khan ZI. Microemulsions: A novel approach to enhanced drug delivery. Recent Pat Drug Del Formul 2008;2:238-57.

8. Naimish AS, Mayur AN, Patel VP. Emerging trend of microemulsion in formulation and research. Int Bull Drug Res 2011;1:54-83.

9. Chavda H, Patel J, Chavada G, Dave S, Patel A, Raval M, et al. Self-nanoemulsifying powder of isotretinoin: Preparation and characterization. J Powder Technol 2013;2013:1-9.

10. Patel MR, Patel RB, Parikh JR, Solanki AB, Patel BG. Effect of formulation components on the in vitro permeation of microemulsion drug delivery system of fluconazole. AAPS Pharm Sci Tech 2009;10:917-23.

11. Ahammed SN, Srinivasan S, Kavitha K, Kumar MR, Kumar PS. A review on microemulsions. Int J Curr Pharm Res 2013:5:10-4

12. Pawar N, Chaudhary H. Non-pressurized topical spray of diclofenac diethylamine. Int J Adv Pharm 2015;4:40-8.

13. Muller RH. Colloidal carriers for controlled drug delivery and targeting. Modification, characterization, and in vivo distribution. In: Wissenschaftliche Verlagsgesellschaft $\mathrm{GmbH}$. Boston: CRC Press; 1991. p. 540-6.

14. Malvern Instruments. New Dynamic Light Scattering Technology for High Sensitivity and Measurement at High Concentration (NIBS). McGraw Hill: Malvern Instruments Ltd; 2008.

15. Instruments M. Zeta Potential Theory. UK: Malvern Instruments Ltd; 2008.

16. Haigh J. The selection and use of natural and synthetic membranes for in vitro diffusion experiments. Eur J Pharm Sci 1994;2:311-30.

17. Patel MR, Patel RB, Parikh JR, Patel BG. improving the isotretinoin photostability by incorporating in microemulsion matrix. ISRN Pharm 2011;2011:1-6

18. Ioele G, Cione E, Risoli A, Genchi G, Ragno G. Accelerated photostability study of tretinoin and isotretinoin in liposome formulations. Int J Pharm 2008;293:251-60. 